\title{
Parâmetros e tendências genéticas para características de crescimento em bovinos da raça Nelore no Estado da Bahia utilizando inferência bayesiana
}

\author{
Parameters and genetic trends for growth traits of Nellore cattle from Bahia state using \\ bayesian inference
}

\section{LACERDA, Júlio Jaat Dias ${ }^{1}$; CARNEIRO, Paulo Luiz Souza ${ }^{2}$; MARTINS FILHO, Raimundo ${ }^{3}$; MALHADO, Carlos Herinque Mendes ${ }^{2}$}

\begin{abstract}
${ }^{1}$ Universidade Estadual do Sudoeste da Bahia, Itapetinga, Bahia, Brasil.
${ }^{2}$ Universidade Estadual do Sudoeste da Bahia, Departamento de Ciências Biológicas, Jequié, Bahia, Brasil.

${ }^{3}$ Universidade Federal do Cariri, Campus de Juazeiro do Norte, Ceará, Brasil.

*Endereço para correspondência: juliojaatlacerda@hotmail.com
\end{abstract}

\section{RESUMO}

O objetivo com esta pesquisa foi estimar parâmetros genéticos e predizer tendências genéticas e fenotípicas para pesos aos 205 (P205), 365 (P365) e 550 (P550) dias de idade, em animais da raça Nelore nascidos no período de 1975 a 2011 no Estado da Bahia. Os dados foram analisados através de modelos bayesianos uni e bicaracterísticos com o programa GIBBS3F90. Os modelos utilizados para as três características incluíram os efeitos aleatórios genéticos (direto e materno), de ambiente permanente maternal, a covariável idade da vaca ao parto (efeito linear e quadrático), além do efeito fixo de grupo de contemporâneos. As herdabilidades (direta) para P205, P365 e P550 apresentaram valores inferiores aos relatados na literatura, contudo, existe possibilidade de ganho genético por meio da seleção. As predições de tendências genéticas e fenotípicas para as características foram obtidas pela regressão linear ponderada da média da variável dependente (valores genéticos e pesos observados) sobre o ano de nascimento. As tendências para P365 e P550 preditas demonstram que houve algum progresso genético para estas duas características, porém, inferiores ao peso aos 205 de idade. Os valores observados indicam pequeno, porém, favorável progresso genético para $\mathrm{o}$ efeito direto nas três pesagens. $\mathrm{O}$ progresso fenotípico foi alto nas três características, mostrando melhoria ambiental, no estado da Bahia, no decorrer do período estudado.

Palavras-chave: correlação genética, ganho genético, herdabilidade, seleção

\section{SUMMARY}

The aim of this study was to estimate genetic parameters and genetic and phenotypic trends for the weights at, 205 (P205), 365 (P365) and 550 (P550) days of age in Nellore cattle born between 1975 to 2011 from Bahia state. Data were analyzed using Bayesian uni-traits and bitraits models with the GIBBS3F90 software. The model used for all traits included the direct and maternal genetic effects, maternal permanent environment effect, the covariate age at calving (linear and quadratic effects), plus the fixed effect of contemporary group. The heritability (direct) for the three characteristics, P205, P365 and P550 were lower than those reported in the literature, however, there is possibility of genetic gain through selection. Estimates of genetic and phenotypic trends for traits were obtained by linear regression weighted average of the dependent variable (breeding value and weights) on the year of birth. Trends estimated for P365 and P550 show that there was some genetic progress for these two traits, however, lower the weight to age 205. The observed values indicate small but favorable genetic progress for direct effect in three traits. Phenotypic progress was high in all traits, showing environmental improvement throughout the study period in Bahia state.

Keywords: genetic correlation, genetic gain, heritability, selection 


\section{INTRODUÇÃO}

A bovinocultura da região Nordeste do Brasil é formada basicamente de animais zebus ou azebuados criados a pasto, principalmente por sua adaptabilidade às condições edafo-climáticas da região, sendo representados, principalmente, pela raça Nelore. Como consequência de secas periódicas, baixo potencial genético dos animais e das pastagens, a produção e a produtividade são baixas, impondo prejuízos econômicos à atividade $\mathrm{e}$ limitando a implementação de programas de melhoramento genético (HOLANDA et al., 2004; MALHADO et al., 2005a).

Nas duas últimas décadas, vários programas de melhoramento foram implementados no Brasil para várias raças bovinas de corte, incluindo diversas características nas avaliações genéticas. $\mathrm{O}$ peso, avaliado em diferentes idades, é uma característica de crescimento largamente utilizada como critério de seleção nesses programas de melhoramento.

Visando buscar eficiência produtiva, com base na genética, um programa de melhoramento genético animal, objetiva identificar animais geneticamente superiores, realizar seleção e acompanhar as mudanças genéticas, com o objetivo de orientar decisões futuras. Para isso é necessário a estimativa dos componentes de (co) variância para a obtenção dos parâmetros genéticos e valores genéticos. A escolha dos métodos utilizados na estimação dos componentes de (co) variância e parâmetros genéticos são fundamentais nas avaliações genéticas. Recentemente, a abordagem Bayesiana vem sendo utilizada como alternativa para a estimativa dos componentes de (co) variância e avaliação genética, podendo ser usada em pequenos ou grandes conjuntos de dados, e em modelos mais complexos, não sendo necessário o conhecimento da distribuição inicial do parâmetro que se deseja estimar. Os métodos de Monte Carlo baseados em cadeias de Markov (MCMC), dentre os quais se destaca o Amostrador de Gibbs, têm sido aplicados na obtenção das distribuições marginais dos parâmetros de interesse de forma iterativa. A grande vantagem deste método é a possibilidade de construção de intervalos de confiança para as estimativas dos componentes de (co) variância, fornecendo uma descrição mais completa sobre a confiabilidade dos parâmetros genéticos (GIANOLA \& FERNANDO, 1986).

Assim, objetivou-se com este estudo estimar parâmetros genéticos e predizer tendências genéticas e fenotípicas para pesos ajustados aos 205; 365 e 550 dias de idade em bovinos da raça Nelore no Estado da Bahia, usando inferência Bayesiana.

\section{MATERIAL E MÉTODOS}

Os dados utilizados foram provenientes do controle de desenvolvimento ponderal de bovinos da Raça Nelore, nascidos entre 1975 a 2011, criados exclusivamente a pasto no estado da Bahia. O banco de dados inicial continha 56.882 animais, com 41.625, 26.167 e 21.761 registros para P205, P365 e P550 dias de idade (Tabela 1), respectivamente.

Os componentes de (co) variância foram estimados mediante análises bayesianas uni e bicaracterísticas, utilizando os softwares GIBBS3F90 (MISTAL, 2012).

As fontes de variação consideradas para a formação dos grupos de contemporâneos (GC) foram: sexo, ano, época de nascimento e fazenda. As épocas de nascimento foram agrupadas 
em quatro classes de forma trimestral (época 1: maio, junho e julho; época 2: agosto, setembro e outubro; época 3 : novembro, dezembro e janeiro; e época
4: fevereiro, março e abril). Grupos de contemporâneos com menos de quatro animais ou desconectados foram excluídos das análises.

Tabela 1. Número de observações, número de grupos contemporâneos (GC) e estatísticas descritivas para os pesos ajustados aos 205 (P205), 365 (P365) e 550 (P550) dias de idade, em bovinos da raça Nelore no Estado da Bahia

\begin{tabular}{lccccc}
\hline Características & $\mathrm{N}^{0}$ de observações & $\mathrm{N}^{\mathrm{o}} \mathrm{GC}$ & Média & Desvio Padrão & $\mathrm{CV}(\%)^{1}$ \\
\hline P205 & 41625 & 3669 & 173,66 & 37,77 & 21,74 \\
P365 & 26167 & 2345 & 238,10 & 60,10 & 25,24 \\
P550 & 21761 & 1952 & 311,38 & 90,25 & 28,98 \\
\hline
\end{tabular}

${ }^{1} \mathrm{CV}=$ Coeficiente de Variação; $\mathrm{GC}=$ Grupo de Contemporâneo.

Os modelos utilizados incluíram os efeitos aleatórios genéticos (direto e materno), de ambiente permanente materno, a covariável idade da vaca ao parto (efeito linear e quadrático), além do efeito fixo de grupo de contemporâneos. Matricialmente $\mathrm{o}$ modelo para P205, P365 e P550 foi descrito da seguinte maneira:

$y=X B+Z a+Z m+Z c+e$

Em que $y=$ vetor de observações; $B=$ vetor de efeitos fixos de GC e covariável idade da vaca ao parto (efeito linear e quadrático); $X=$ matriz de incidência que relaciona as observações aos efeitos fixos; $a=$ vetor dos efeitos aleatórios aditivo direto, $m=$ vetor dos efeitos aleatórios aditivo materno; $c=$ vetor dos efeitos aleatórios de ambiente permanente materno; $e=$ vetor dos erros aleatórios; $Z=$ matriz de incidência que relaciona os efeitos aleatórios ao vetor de observações.

As densidades marginais dos componentes de (co) variância genéticas foram estimadas pelas amostras geradas pelos métodos de Monte Carlo via Cadeias de Markov (MCMC) usando o Amostrador de Gibbs. Adotando-se os seguintes procedimentos: a) Rodou-se uma amostra piloto com 100.000 ciclos, 10.000 de descarte (burn-in) e 10 para o intervalo de salvamento (thin); b) utilizou-se o arquivo resposta 'postgibbs_samples', e o pacote BOA (Bayesian Output Analysis) do programa R (R DEVELOPMENT CORE TEAM, 2008), aplicando o teste Raftery \& Lewis (1992) para determinar o novo tamanho da cadeia, "burn-in" e o "thin" (Tabela 2). O diagnóstico de convergênciafoi realizado seguindo o método Geweke (1992).

Tabela 2. Definição do número total de iterações, descarte ("burn-in") e salvamento ("thin") para as características pesos aos 205 (P205), 365 (P365) e 550 (P550) dias de idade após teste de convergência

\begin{tabular}{lccc}
\hline Características & Cadeia total & Burn-in & Thin \\
\hline P205 & 800.000 & 80.000 & 10 \\
P365 & 700.000 & 70.000 & 70 \\
P550 & 600.000 & 60.000 & 40 \\
\hline
\end{tabular}


Rev. Bras. Saúde Prod. Anim., Salvador, v.15, n.1, p.10-19 jan./mar., 2014 http://www.rbspa.ufba.br ISSN 15199940

As estimativas das tendências genéticas (diretas e maternas) e fenotípicas para as características avaliadas foram obtidas pela regressão linear ponderada da média da variável dependente (valores genéticos e pesos observados) sobre o ano de nascimento.

\section{RESULTADOS E DISCUSSÃO}

A estimativa de herdabilidade direta $\left(\mathrm{h}^{2} \mathrm{~d}\right)$ foi de média magnitude $(0,21 \pm 0,02)$ para o peso aos 205 dias de idade (P205) (Tabela 3). Lira et al. (2008), em uma revisão com base em 67 trabalhos com zebuínos, encontraram estimativa média de $\mathrm{h}^{2} \mathrm{~d}$ de 0,28. Malhado et al. (2005a) relataram $h^{2} d$ de 0,24 na raça Nelore mocho no estado da Bahia.

Para P205, observou-se baixa herdabilidade materna $(0,06 \pm 0,02)$, indicando baixa influência dos efeitos maternos para essa característica. Valores superiores foram encontrados por Malhado et al. (2010) $(0,12)$ e Malhado et al. (2005a) $(0,20)$ na raça Nelore e Nelore Mocho, respectivamente.

Tabela 3. Estatísticas descritivas dos parâmetros genéticos para os pesos aos 205 (P205), 365 (P365) e 550 (P550) dias de idade, em bovinos da raça Nelore, no Estado da Bahia

\begin{tabular}{lccc}
\hline \multicolumn{4}{c}{ Parâmetros Genéticos P205 } \\
\hline Estimativas & $\mathrm{h}^{2} \mathrm{~d}$ & $\mathrm{~h}^{2} \mathrm{~m}$ & $\mathrm{rGam}$ \\
\hline Média & 0,21 & 0,06 & $-0,25$ \\
Mediana & 0,20 & 0,06 & $-0,26$ \\
Moda & 0,21 & 0,06 & $-0,44$ \\
DP & 0,01 & 0,02 & 0,12 \\
IC $(95 \%)$ & 0,17 a 0,24 & 0,01 a 0,10 & $-0,49$ a 0,00 \\
\hline \multicolumn{4}{c}{ Parâmetros Genéticos P365 } \\
\hline Estimativas & $\mathrm{h}^{2} \mathrm{~d}$ & $\mathrm{~h}^{2} \mathrm{~m}$ & $\mathrm{rGam}$ \\
\hline Média & 0,21 & 0,08 & $-0,006$ \\
Mediana & 0,21 & 0,08 & $-0,01$ \\
Moda & 0,19 & 0,04 & 0,20 \\
DP & 0,02 & 0,03 & 0,20 \\
IC (95\%) & $0,15 \mathrm{a} 0,26$ & $0,02 \mathrm{a} 0,14$ & $-0,40 \mathrm{a} 0,37$ \\
\hline \multicolumn{4}{c}{ Parâmetros Genéticos P550 } \\
\hline Estimativas & $\mathrm{h}^{2} \mathrm{~d}$ & $\mathrm{~h}^{2} \mathrm{~m}$ & $\mathrm{rGam}$ \\
\hline Média & 0,19 & 0,06 & $-0,35$ \\
Mediana & 0,19 & 0,06 & $-0,37$ \\
Moda & 0,17 & 0,03 & - \\
DP & 0,02 & 0,03 & 0,18 \\
IC (95\%) & $0,15 \mathrm{a} 0,24$ & $0,01 \mathrm{a} 0,10$ & $-0,72 \mathrm{a} 0,00$ \\
\hline
\end{tabular}

$\mathrm{h}^{2} \mathrm{~d}=$ herdabilidade direta; $\mathrm{h}^{2} \mathrm{~m}=$ herdabilidade materna; $\mathrm{rGam}=$ correlação genética entre os efeitos direto e materno; DP = Desvio Padrão; IC = Intervalo de credibilidade a 95\%.

A estimativa da herdabilidade direta para P365 também foi igual a 0,21. Este valor é menor que os relatados por Faria et al. (2008), Malhado et al. (2010) e
Boligon et al. (2008), que estimaram valores de 0,39, 0,37 e 0,30, respectivamente, estudando animais da raça Nelore. A estimativa da 
herdabilidade materna para P365 foi $(0,08 \pm 0,03)$, superior as estimativas relatadas por Bertazzo et al. (2004) $(0,03)$ e Ferraz Filho et al. (2002) $(0,03)$ em bovinos da raça Nelore. De acordo com Bertazzo et al. (2004) as estimativas de $h^{2} \mathrm{~m}$ para P365 encontradas na literatura, variaram de 0,07 a 0,27 .

As estimativas de $h^{2} d$ para a característica P550 foi de 0,19, inferior aos relatados por Gianotti et al. (2005), Bertazzo et al. (2004) e Boligon et al. (2008), que encontraram valores de $h^{2} d$ iguais a $0,31,0,35$, e 0,30 , respectivamente. A estimativa da $h^{2} \mathrm{~m}$ para P550 obtida neste trabalho foi igual a $(0,06 \pm 0,03)$ semelhante $a h^{2} m$ de P205. Valores baixos de $h^{2} \mathrm{~m}$, relatados neste trabalho, podem ser explicados pelo fato de que os efeitos maternos tiveram pouca influência sobre os pesos.

Os coeficientes de herdabilidades (direta) para as três características apresentaram valores de baixas a médias magnitudes, o que indica haver variabilidade genética aditiva direta no Nelore do Estado da Bahia, evidenciando oportunidade de ganho genético com a seleção para peso nesta raça, mostrando também a necessidade de se utilizar informações proporcionadas por parentes no processo de seleção.

As correlações genéticas entre os efeitos aditivos diretos e maternos (rGam) foram baixas e negativas, com valores de $\quad-0,25 \pm 0,12 \quad(\mathrm{P} 205), \quad-0,006 \pm 0,20$ (P365) e $-0,35 \pm 0,18$ (P550). Os erros padrões associados às estimativas indicam que a estrutura dos dados podem ter sido insuficientes para uma estimativa com menor intervalo de credibilidade deste parâmetro (Tabela 3 ). Boligon et al. (2012), discutiram que problemas na estruturação de dados, como por exemplo, pedigree com pouca profundidade, pode ocasionar estimativas tendenciosas para os componentes de (co) variância, com estimativa negativa para a covariância entre os efeitos genéticos direto $\mathrm{e}$ mateno.

Para a característica P205, a tendência genética (regressão linear) de efeito direto foi significativa $(\mathrm{p}<0,0001)$ e igual a $0,0634 \mathrm{~kg} / \mathrm{ano}$, que equivale a $2,28 \mathrm{~kg}$ durante os 36 anos avaliados (Figura 1). Em termos de mudança genética anual, este resultado representa um ganho de 0,036\% por ano. Essa tendência foi superior a encontrada por Malhado et al. (2005b), estudando bovinos da raça Nelore Mocho no Estado da Bahia. Estes autores relataram tendência genética direta negativa $(\mathrm{p}<0,05)$ para $\mathrm{P} 205$, com valor estimado de $-0,07 \mathrm{~kg} / \mathrm{ano}$. Holanda et al. (2004) estimaram tendência genética direta negativa de $-0,02 \mathrm{~kg} /$ ano para $\mathrm{o}$ efeito direto em bovinos Nelore em Pernambuco.

Observou-se um progresso genético crescente, a partir de 1997 (Figura 1), evidenciando que nos últimos anos existe em alguns rebanhos seleção para o peso ao desmame. Esse progresso apesar de pequeno é importante, pois as mudanças são estáveis e cumulativas ao longo dos anos.

$\mathrm{Na}$ característica P365, a tendência genética direta (regressão linear) foi significativa $(p<0,05)$ e igual a $0,0328 \mathrm{~kg} / \mathrm{ano}$, que equivale a $1,14 \mathrm{~kg}$ durante os 35 anos estudados. Em termos de mudança genética anual, este resultado representa um ganho de $0,01 \%$ por ano (Figura 2). Esta tendência foi superior a encontrado por Val et al. (2008), que relataram ganho inferior ao observado neste estudo $(-1,21 \mathrm{~kg} / \mathrm{ano})$ em bovinos da raça Nelore. 
Rev. Bras. Saúde Prod. Anim., Salvador, v.15, n.1, p.10-19 jan./mar., 2014 http://www.rbspa.ufba.br ISSN 15199940

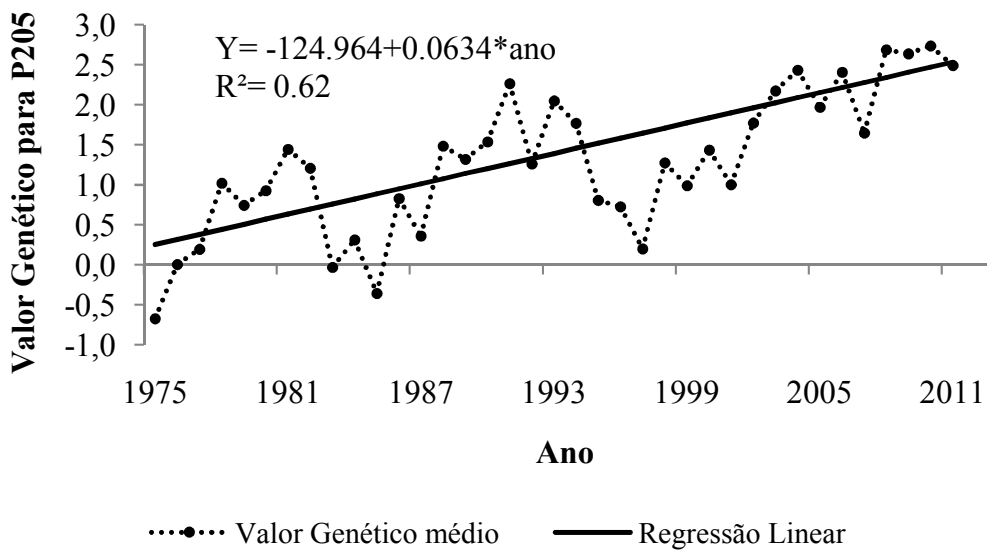

Figura 1. Tendência genética para a característica P205 em bovinos da raça Nelore no Estado da Bahia, no período de 1975 a 2011

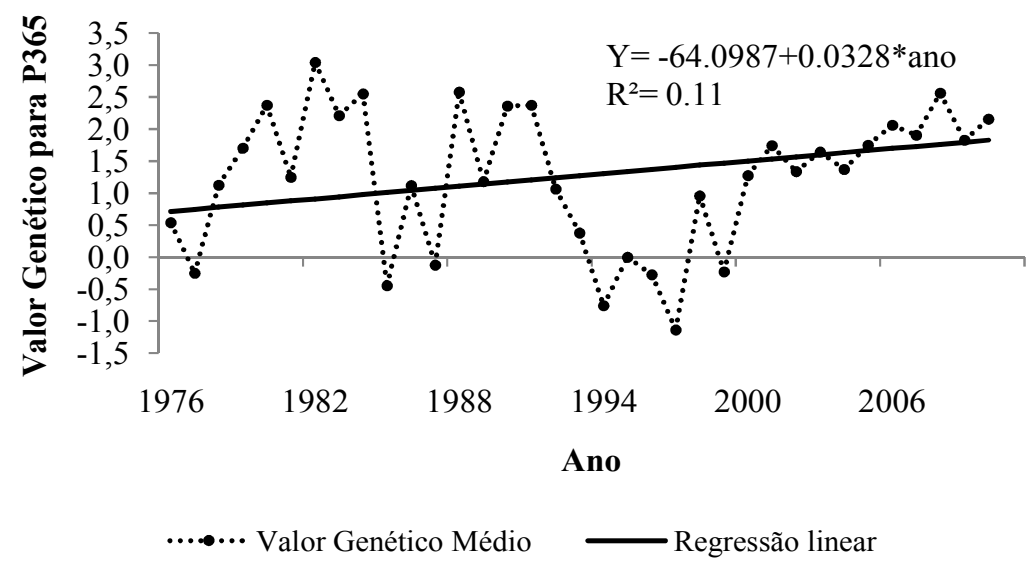

Figura 2. Tendência genética para a característica P365 em bovinos da raça Nelore noEstado da Bahia, no período de 1976 a 2010

A tendência genética direta (regressão linear) para P550 foi significativa $(\mathrm{p}<0,001)$ com valor estimado de $0,0489 \mathrm{~kg} / \mathrm{ano}$, que equivale a $1,71 \mathrm{~kg}$ durante os 35 anos estudados. Se tratando de mudança genética anual corresponde um ganho de $0,01 \%$ (Figura 3). Malhado et al. (2008) apresentaram valores positivos $(0,068 \mathrm{~kg} / \mathrm{ano})$ para a tendência genética do peso aos 550 dias de idade. Por outro lado, resultados negativos foram estimados por Val et al. (2008) em bovinos da raça Nelore, os quais relataram valor de $-0,80 \mathrm{~kg} / \mathrm{ano}$.

As tendências para P365 e P550 estimadas no presente trabalho demonstram que houve algum progresso genético para estas duas características, porém, inferiores ao peso aos 205 de idade. Desta forma, evidencia-se que os criadores que fazem seleção, estão utilizando mais o peso aos 205 dias de idade como critério de seleção do que as outras duas pesagens. 
Rev. Bras. Saúde Prod. Anim., Salvador, v.15, n.1, p.10-19 jan./mar., 2014 http://www.rbspa.ufba.br ISSN 15199940

Os ganhos genéticos foram baixos, sugerindo pequena intensidade de seleção para essa raça no estado. Contudo, devemos salientar que, provavelmente, existam rebanhos com forte seleção para estas características na Bahia. Estes resultados estão de acordo com parte da literatura científica, que mostram respostas genéticas relativamente baixas para características de crescimento em animais de raças zebuínas.

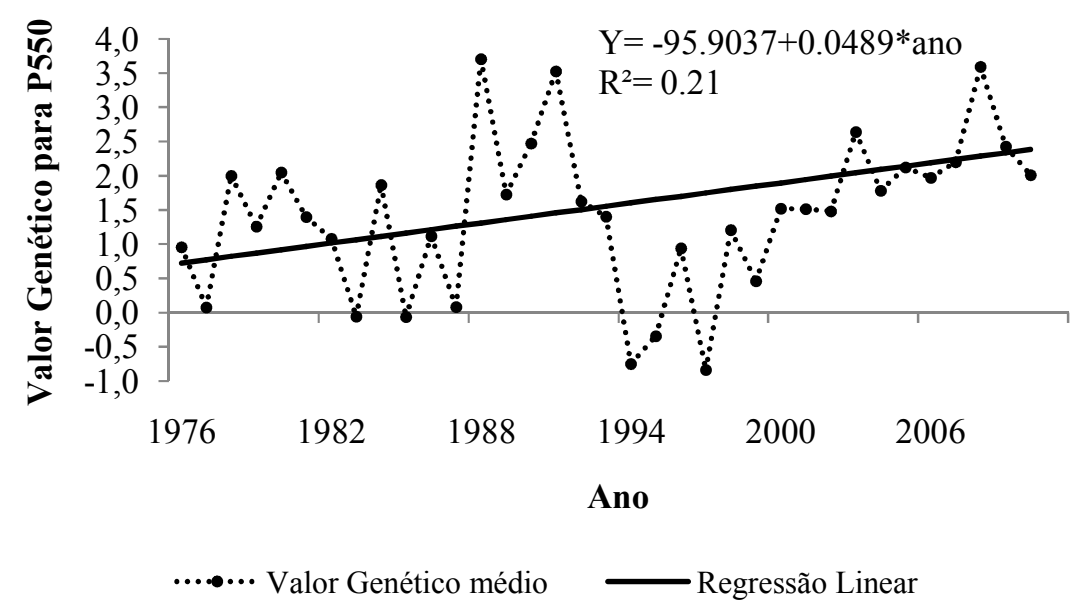

Figura 3. Tendência genética para a característica P550 em bovinos da raça Nelore noEstado da Bahia, no período de 1976 a 2010

Durante os 36 anos estudados, as tendências maternas foram negativas para as três características, com decréscimos anuais de $-0,0187 \mathrm{~kg} / \mathrm{ano}$ (P205), -0,0044kg/ano (P365) e $0,0262 \mathrm{~kg} / \mathrm{ano}(\mathrm{P} 550)$. Estas estimativas negativas podem ser consequência da correlação genética negativa entre os efeitos direto e materno, decorrente da estrutura dos dados. Contudo, não descartamos a possibilidade de ausência de seleção para as características maternas. Valores negativos para as tendências genéticas maternas são comuns na literatura, Malhado et al. (2005b) encontraram valor de 0,02kg/ano, para P205 dias, em bovinos da raça Nelore Mocho no estado da Bahia. Ferraz Filho et al. (2002) verificaram tendências negativas para $\mathrm{o}$ efeito materno para P365 $(-0,01 \mathrm{~kg} / \mathrm{ano})$ e P550 (-0,02kg/ ano), representando perdas anuais de 0,005 e 0,007\% em relação à media para os pesos aos $365 \mathrm{e}$ 550 dias de idade, respectivamente.

As tendências fenotípicas refletem $o$ progresso da característica na sua própria unidade de medida, possibilitando observar se os programas de seleção conjuntamente com melhorias ambientais tem sido favoráveis a produção ao longo do tempo. As tendências fenotípicas para as características P205, P365 e P550 foram significativas $(p<0,001) \quad \mathrm{e}$ respectivamente iguais a $1,13 \mathrm{~kg} / \mathrm{ano}$; $1,25 \mathrm{~kg} /$ ano e 1,69kg/ano (Figuras 4, 5 e 6), o que representa incrementos de 40,$68 ; 45,00$ e $61,20 \mathrm{~kg}$, respectivamente para os pesos durante o período analisado (36 anos). Em termos de mudança fenotípica anual, estes valores representam em relação à média para os pesos aos P205; P365 e P550, ganhos de $0,65 \% ; 0,52 \%$ e $0,54 \%$ respectivamente. 
Rev. Bras. Saúde Prod. Anim., Salvador, v.15, n.1, p.10-19 jan./mar., 2014 http://www.rbspa.ufba.br ISSN 15199940

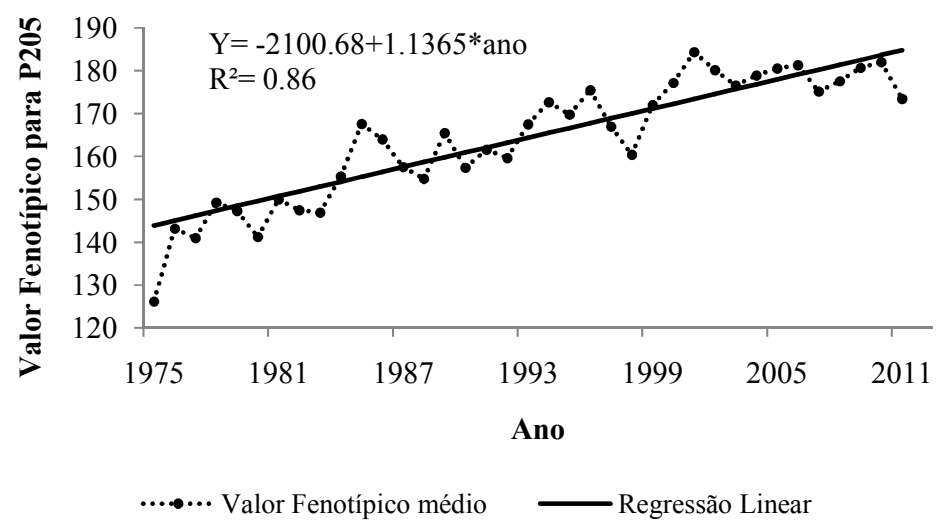

Figura 4. Tendência fenotípica para a característica P205 em bovinos da raça Nelore noEstado da Bahia, no período de 1975 a 2011

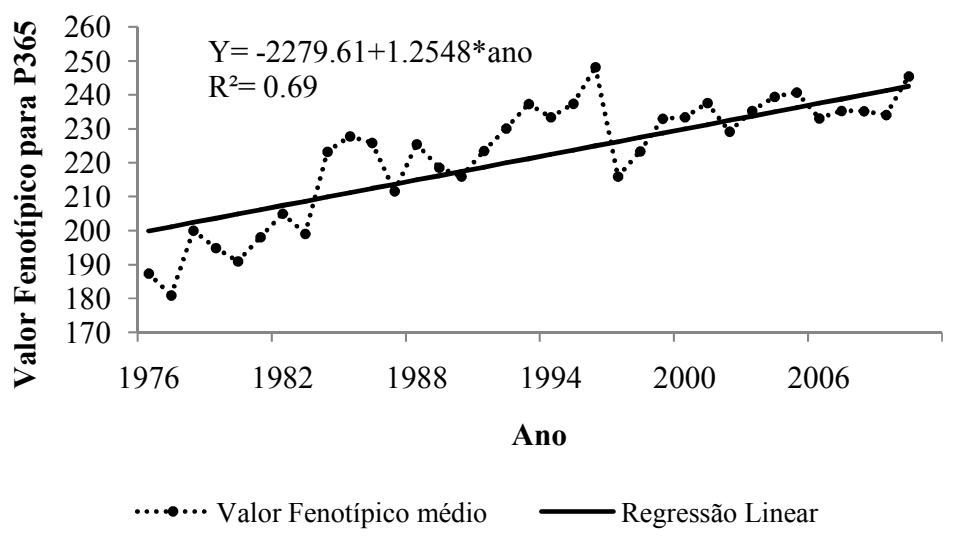

Figura 5. Tendência fenotípica para a característica P365 em bovinos da raça Nelore no Estado da Bahia, no período de 1976 a 2010

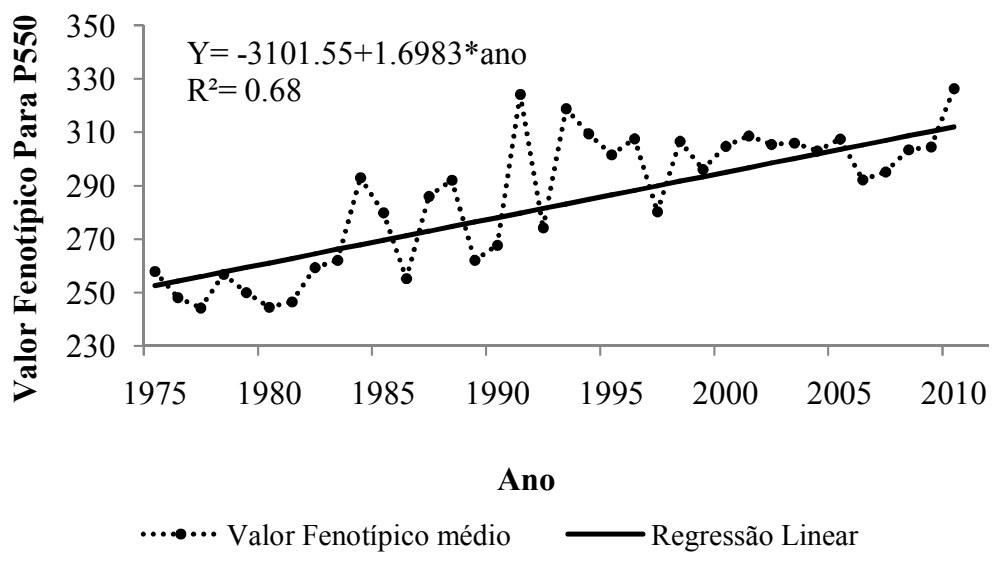

Figura 6. Tendência fenotípica para a característica P550 em bovinos da raça Nelore noEstado da Bahia, no período de 1975 a 2010 
Rev. Bras. Saúde Prod. Anim., Salvador, v.15, n.1, p.10-19 jan./mar., 2014 http://www.rbspa.ufba.br ISSN 15199940

As estimativas dos coeficientes de herdabilidades indicam existência de variabilidade genética aditiva direta no Nelore do estado da Bahia, evidenciando oportunidade de ganho genético com a seleção.

Os valores obtidos para a tendência genética direta para as três características são baixos. As tendências fenotípicas mostram que as características estão melhorando com o tempo, ganhos atribuídos, em quase sua totalidade, as melhorias no ambiente.

\section{AGRADECIMENTOS}

À Universidade Estadual do Sudoeste da Bahia; à Coordenação de Aperfeiçoamento de Pessoal de Nivel Superior, pela bolsa concedida ao primeiro autor, e à Associação Brasileira de Criadores de Zebu, pelo acesso dos dados.

\section{REFERÊNCIAS}

BERTAZZO, R.P.; FREITAS, R.T.F.; GONÇALVES, T.M.; PEREIRA, I.G.; ELER, J.P.; FERRAZ, J.B.S.;

OLIVEIRA, A.I.G.; ANDRADE, I.F.

Genetic parameters of longevity and productivity of Nellore females..

Revista Brasileira de Zootecnia, v.33, p.1118-1127, 2004.

BOLIGON, A.A.; ALBUQUERQUE, L.G.; RORATO, P.R.N. Genetic associations among weights and reproductive traits in Nellore cattle.

Revista Brasileira de Zootecnia, v.37, p.596-601, 2008.

BOLIGON, A.A.; PEREIRA, R. J.; AYRES, D.R.; ALBURQUEQUE, L.G. Influence of datstruture on the estimation of the additive genetic direct and maternal covariance for early growth traits in Nellore cattle.

Livestock Science, v.145, p.212-218, 2012.

FARIA, C.U.; MAGNABOSCO, C.U.; ALBURQUEQUE, L.G.; REYES, A.L.; BEZERRA, L.A.F.; LÔBO, R.B.

Bayesian and frequentist approaches in three trait genetic analysis for growth and reproduction in nelore cattle.

Ciência Animal Brasileira, v.9, n.3, p.598-607, 2008.

FERRAZ FILHO, P.B.; RAMOS, A.A.; SILVA, L.O.C.; SOUZA, J.C.; ALENCAR, M.M.; MALHADO, C.H.M. Genetic Trends of the Direct and Maternal Effects for Weaning and Post-Weaning Weights of Tabapuã Cattle in Brazil. Revista Brasileira de Zootecnia, v.31, p.635-640, 2002.

GEWEKE, J. Bayesian Statistics, chapter Evaluating the Accuracy of Sampling- Based Approaches to Calculating Posterior Moments. Oxford: Oxford University Press, 1992. v.4.

GIANOLA, D.; FERNANDO, R.L. Bayesian methods in animal breeding theories. Journal Animal Science, v.63, p.217-244, 1986.

GIANNOTTI, J. di G.; PACKER, I.U.; MERCADANTE, M.E.Z. Meta-analysis for heritability of estimates growth traits in beef cattle. Revista Brasileira de Zootecnia, v.34, p.1173-1180, 2005.

HOLANDA, M.C.R.; BARBOSA, S.B.P.; RIBEIRO, A.C.; SANTORO, K.R. Genetic trends for growth in nellore beef cattle in Pernambuco, Brazil. Archivos de Zootecnia, v.53, p.185-194. 2004. 
Rev. Bras. Saúde Prod. Anim., Salvador, v.15, n.1, p.10-19 jan./mar., 2014 http://www.rbspa.ufba.br ISSN 15199940

LIRA, T. de; ROSA, E.M.; GARNERO, A. del V. Genetic parameters and reproductive and productive characteristics in beef cattle revision.

Ciência Animal Brasileira, v.9, p.1-22, 2008.

MALHADO, C.H.M.; CARNEIRO, P.L.S.; MARTINS FILHO, R.; AZEVEDO, D.M.M.R.; FACO, O.; MACHADO, C.H.C.; PICCININ, A. Trend and parameters genetic for weight at 205 day of bovines of Polled Nellore Breed from Bahia State.

Revista Científica de Produção Animal, v.7, p.28-34, 2005a.

MALHADO, C.H.M.; FILHO, R.M.; LÔBO, R.N.B.; FACÓ, O.; AZEVEDO, D.M.P.R.; SOUZA, J.C. de; OLIVEIRA, S.M.P. Genetic trends for characteristics related to the growth rate in Nellore cattle from Northern Brazil.

Revista Brasileira de Zootecnia, v.34, n.1, p.60-65, 2005b.

MALHADO, C.H.M.; CARNEIRO, P.L.S.; PEREIRA, D.G.; MARTINS FILHO, R.; SOUZA, J.C. Genetic progress and population structure in Nellore cattle in Bahia State, Brazil. Pesquisa Agropecuária Brasileira, v.43, p.1163-1169, 2008.

MALHADO, C.H.M.; CARNEIRO, P.L.S.; JAMES, R.P. Genetic improvement and population structure of the Nelore breed in Northern Brazil. Pesquisa Agropecuária Brasileira, v.45, n.10, p.1109-1116, 2010.
MISTAL, J. 2012. Fortran Programs. Disponível em:

http://nce.ads.uga.edu/wiki/doku.php?id $=$ readme.gib bs 3 . Acesso em 13 de setembro 2012.

R DEVELOPMENT CORE TEAM. R: a language and environment for statistical computing. Vienna: $R$ Foundation for Statistical Computing, 2008. Available at: $<$ http://www.Rproject.org $>$. Accessed on: 10 Apr. 2012.

RAFTERY, A.E.; LEWIS S. How many iterations in the Gibbs sampler? In: BERNARDO J.M.; BERGER J.O.; DAWID A.P.; SMITH, A.F.M. (Eds.).

Bayesian statistics 4. Oxford University Press, 1992. p.763-773.

VAL, J.E.; FERRAUDO, A.S.; BEZERRA, L.A.F.; CORRADO, M.P.; LÔBO, R.B.; FREITAS, M.A.R.; PANETO, J.C.C. Nellore bull selection alternatives using multiple traits of economic importance. Arquivo Brasileiro de Medicina Veterinária e Zootecnia, vol.60, n.3, p. 705-712, 2008.

Data de recebimento: 19/07/2013

Data de aprovação: 04/02/2014 